\title{
Inguinal hernia surgery in Korea: nationwide data from 2007-2015
}

\author{
Seung-Rim Han, Hyung-Jin Kim, Nam-Hee Kim, Sukhyun Shin, Ri Na Yoo, Gun Kim, Hyeon-Min Cho \\ Department of Surgery, St. Vincent's Hospital, College of Medicine, The Catholic University of Korea, Suwon, Korea
}

\begin{abstract}
Purpose: The aim of this study is to analyze inguinal hernia in Korea based on the nationwide data.
Methods: Retrospective analysis of nationwide data obtained from the Health Insurance Review \& Assessment Service database for surgical repair of inguinal hernia from January, 2007 to December, 2015 was performed.

Results: A total of 314,238 cases of inguinal hernia surgery were performed during 9 years. The mean number of inguinal hernia surgery per year was 34,604 (range, $30,065-36,928$ ). The proportion of male patient was $87 \%$. Patients between the age of 1 to 5 years $(17.3 \%)$ and of 60 to 69 years $(17.4 \%)$ constituted the 2 dominant groups for inguinal hernia repair. A slight increase in repair for recurrent inguinal hernia, 1.9\% to $3.2 \%$, was observed from 2007 to 2015 . The rate of concurrent bowel resection decreased from $1.9 \%$ to $0.6 \%$. The total cost of inguinal hernia surgery was increased up to 1.68 times from 2007 to 2015 (\$856 to $\$ 1,439$ ), although the number of hospital days was decreased from 4.7 to 3.7 days. The rate of laparoscopic surgery was increased to $29.5 \%$ in 2015.

Conclusion: A mean number of 34,604 cases of inguinal hernia surgery have been carried out annually in Korea. The age distribution for inguinal hernia repair demonstrates bimodal peak at early childhood and old age. The proportion of laparoscopic surgery of inguinal hernia has been increasing for the past 9 years. The length of hospital stay and the rate of combined bowel resection have decreased.
\end{abstract}

[Ann Surg Treat Res 2019;97(1):41-47]

Key Words: Nationwide, Inguinal hernia, Diagnosis-related groups

\section{INTRODUCTION}

Inguinal hernia repair is the most frequently performed surgical procedure in general surgical departments worldwide. According to the Danish database, about 12,000 operations are performed per year [1]. Approximately 800,000 inguinal hernias were repaired in the United States in 2003 [2]. National health data from Ghana in Africa indicate a prevalence of inguinal hernia in the general population of $3.15 \%$ and a hernia repair rate estimate of 30 per 100,000 [3]. However, few studies are available that present epidemiologic data on inguinal hernia repair on a large scale, especially in Asia.

Additionally, since laparoscopic herniorrhaphy was introduced in the early 1990s, studies have compared surgical outcomes between laparoscopic and open hernia surgery. However, few studies are available that show proportions of laparoscopic versus open surgery of inguinal hernia repairs on a nationwide scale.

The present study aimed to investigate the prevalence of inguinal hernia repairs, including femoral hernia, and the rate of laparoscopic inguinal hernia surgery using a nationwide database in Korea. Economic features of inguinal hernia repair
Received November 22, 2018, Revised April 2, 2019,

Accepted April 12, 2019

\section{Corresponding Author: Hyung-Jin Kim}

Department of Surgery, St. Vincent's Hospital, College of Medicine, The Catholic University of Korea, 93 Jungbu-daero, Paldal-gu, Suwon 16247, Korea

Tel: +82-31-249-8316, Fax: +82-31-247-5347

E-mail: hi@catholic.ac.kr

ORCID code: https://orcid.org/0000-0002-6315-714X
Copyright (c) 2019, the Korean Surgical Society

(c) Annals of Surgical Treatment and Research is an Open Access Journal. All articles are distributed under the terms of the Creative Commons Attribution NonCommercial License (http://creativecommons.org/licenses/by-nc/4.0/) which permits unrestricted non-commercial use, distribution, and reproduction in any medium, provided the original work is properly cited. 
were also evaluated based on the Korean medical care system. Further, inguinal hernia repairs along with economic and clinical features were examined for changes brought about by the 2013 adoption of the diagnosis-related group-based (DRGbased) Korean medical insurance system. This system uses prospective payment to replace the fee-for-service system.

\section{METHODS}

Nationwide data on inguinal hernia surgery from 2007 to 2015 were obtained from the Health Insurance Review \& Assessment Service (HIRA) database. Data included information on the year of surgical treatment; sex; age; procedure fees, including total costs and cost-sharing; codes for inguinal hernia surgery, hospital, DRG, and insurance; total hospital days; and type of surgical procedure (laparoscopic or open). Total hospital days were defined as the sum of days spent in the hospital and the number of days for outpatient clinic visits. This study was approved by the Institutional Review Board of St. Vincent Hospital, the Catholic University of Korea as VC17ZESI0092. For this type of study, formal consent is not required.

Data collection procedures were as follows: (1) Data were electronically extracted based on the day of surgery covered by health insurance. The HIRA completed review and assessment of costs for all individual medical services performed from January 2007 and December 2015. (2) Procedure codes were electronically extracted. Inguinal hernia surgical involved various procedures, such as a high ligation with or without posterior wall repair, and recurrent and femoral hernia repair. All procedures can be performed with bowel resection. Thus, procedure fee codes searched were Q2753-56, QA753-56, and Q2757, based on the health insurance medical care benefit cost book (2017 January edition, published by the Korean Hospital Association). (3) Laparoscopic instrument fee codes were extracted. Code N0031001 and DRG code G095-98 were used to identify the number of laparoscopic hernia operations, since no procedure codes for laparoscopic inguinal hernia repair exist. Our data were analyzed only for total values extracted variables. The data cannot be assessed via comparison between groups.

This retrospective analysis was completed with the above data. Changes in the total number of surgical procedures by year were used for comparisons among variables by age group. These groups included children ( 0 to 10 years) and adults ( 20 years or above); teenage patients were not included in the study. Distributions for age, sex, types of insurance, hospitals, total costs, cost-sharing and hospital stay over the nine years of the study were used in the evaluation.

\section{RESULTS}

From 2007 to 2015, a total of 311,441 cases of surgery for

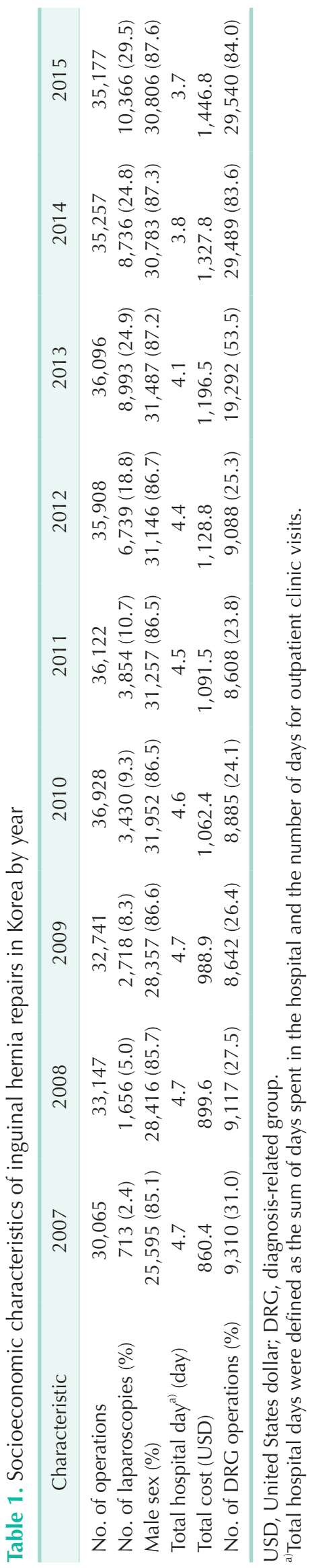


inguinal hernia were performed in Korea (Table 1), comprising 269,799 cases in male (87\%) and 41,642 cases in female patients (13\%). The proportion of males in adult and pediatric patients was $92 \%$ and $75 \%$, respectively. Annually, an average of 34,604 inguinal hernia repairs, either by open or laparoscopy, were performed. Laparoscopic inguinal hernia repairs were dramatically increased between 2007 and 2015, 2.4\% to 29.5\%, while the total number of inguinal hernia operations remained relatively constant (Fig. 1A). The total number of inguinal hernia surgeries for pediatric patients decreased from 2010 onward; however, the proportion of laparoscopic surgery and DRG increased (Fig. 1C). Bimodal peaks of prevalence in children aged 1-5 years (17.3\%) and elderly patients aged 50-59 (12.8\%), 60-69 years (17.3\%), and $70-79$ years $(15.6 \%)$ were observed (Fig. 2).

Medical fees increased every year ( $\$ 860$ in 2007 to $\$ 1,446$ in 2015) (Fig. 3) and DRG-based payments increased to $83 \%$ of total inguinal surgery after the Korean medical charge system was changed completely to DRG in June, 2013. However, hospital stay length slightly decreased from 4.7 days to 3.7 days during the 9 years of the study (Fig. 4). Hospital days after inguinal
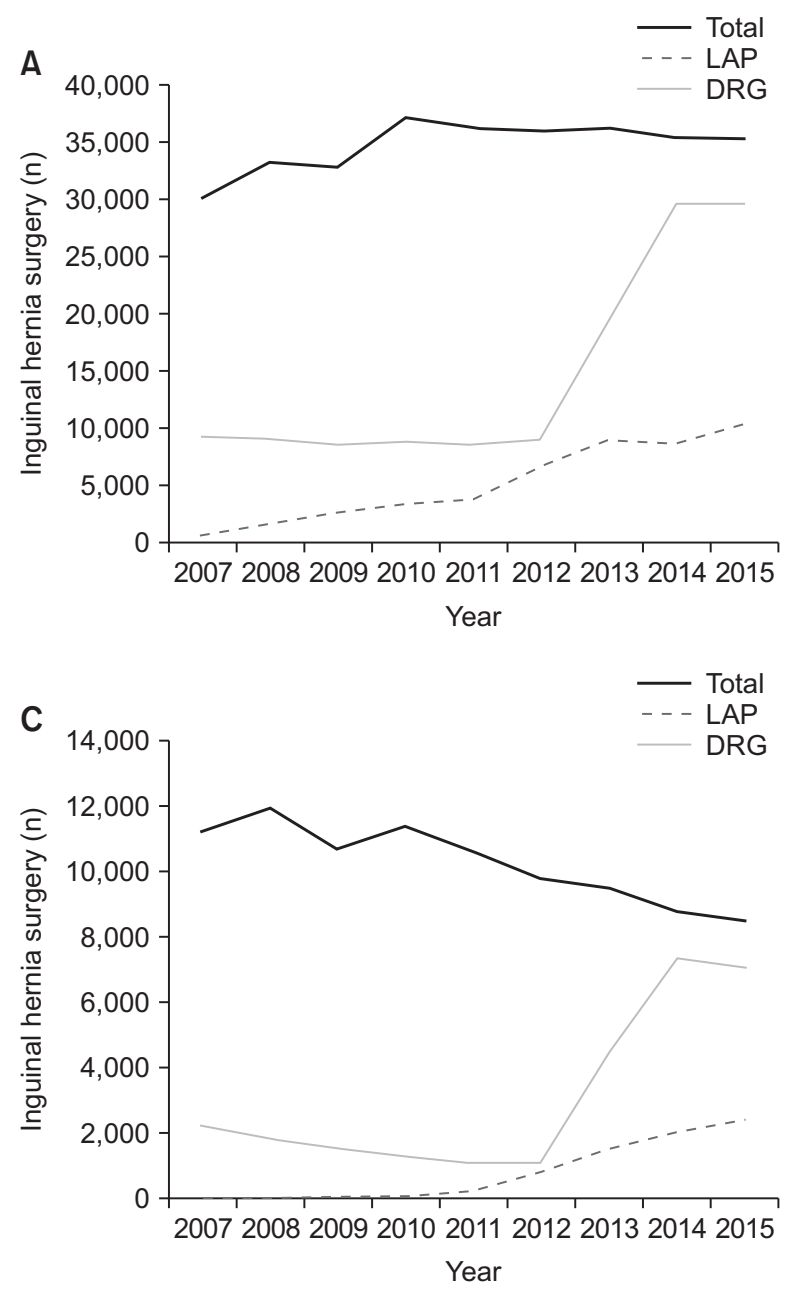

hernia repair decreased regardless of age. Total hospital stay for adult patients was longer than for pediatric patients for every year (Fig. 4). Adult stays were 5.7 days on average in 2007 which

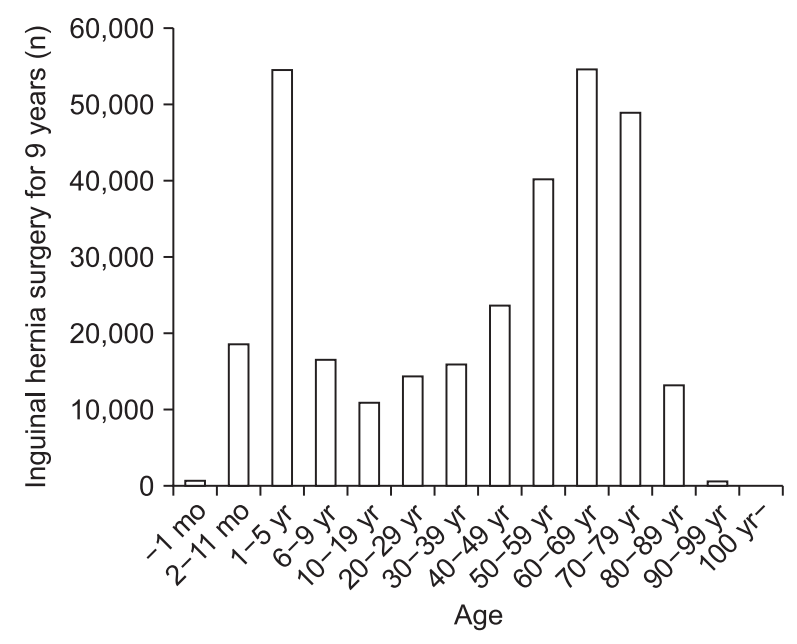

Fig. 2. Distribution of inguinal hernia repairs according to age in Korea for 9 years.

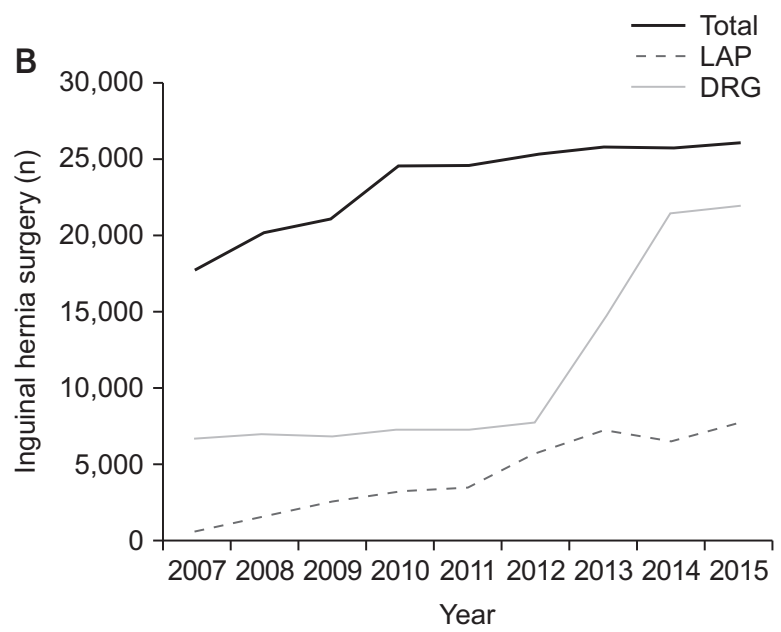

Fig. 1. Trend of proportion of laparoscopic (LAP) and diagnosis-related group (DRG) based inguinal hernia repairs: (A) total inguinal hernia repairs, (B) adult group ( $>20$ years), (C) pediatric group $(<10$ years). 
decreased to 4 days in 2015. Children remained in the hospital for an average of 3.2 days in 2007 and for three days in 2015.

About $75 \%$ of inguinal hernia repairs were performed in general (45\%) and tertiary referral hospitals (30\%) (Fig. 5A). The proportion of operating hospitals varied depending on age subgroups. About half of adult inguinal hernia operations were

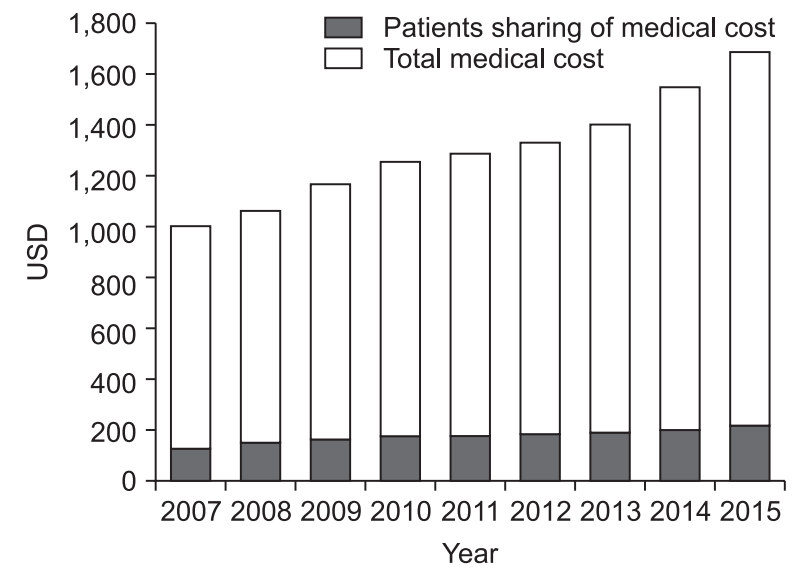

Fig. 3. Trend of total medical cost (United States dollar, USD) of inguinal hernia repair by year. performed in general hospitals, while $49 \%$ of pediatric inguinal hernia repairs were performed in tertiary referral hospitals (Fig. $5 B, C)$.

The composition of hernia procedures was different

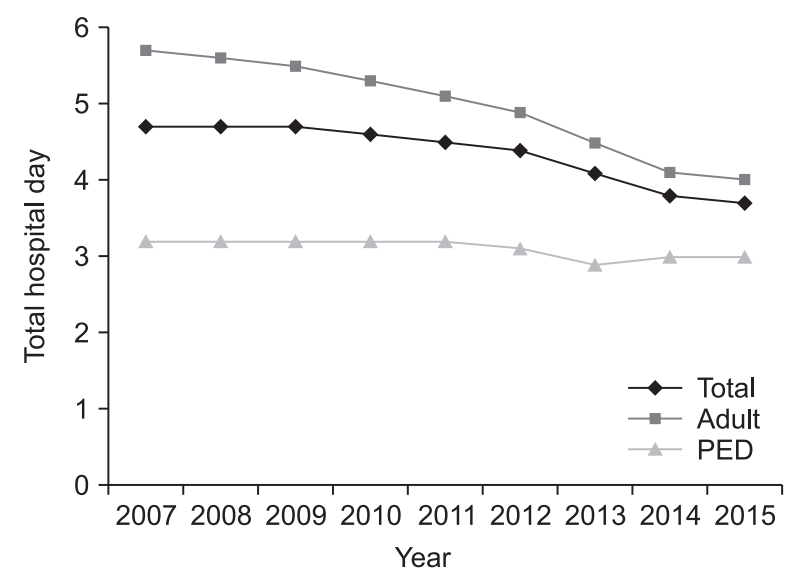

Fig. 4. Trend of total hospital day of inguinal hernia repair by year. Total hospital day is defined by the day of hospitalization and the day of visiting in outpatient clinic. PED, pediatric patient.
A

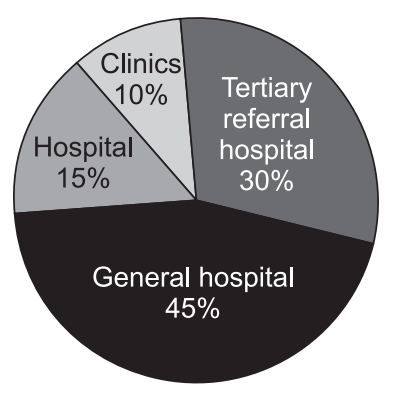

Public health

B

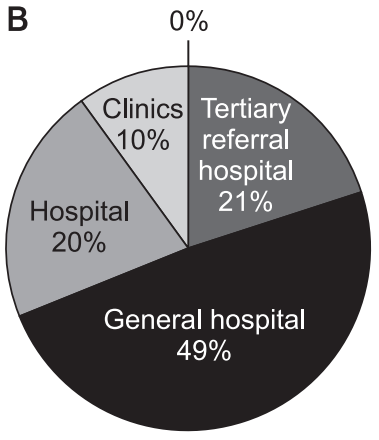

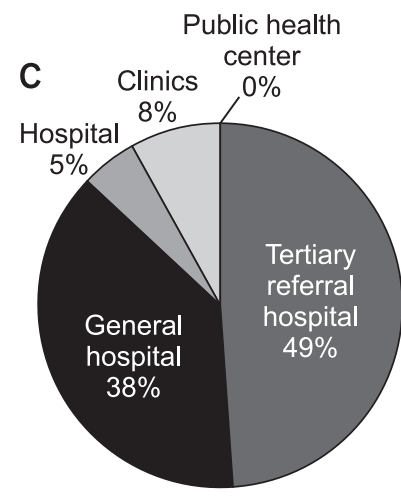

Fig. 5. Distribution of medical providers performing inguinal hernia repairs: (A) total inguinal hernia repairs, (B) adult group, and (C) pediatric group.
Hernia repair with bowel resection High ligation

High ligation with posterior repair

Recurrent hernia repair

Femoral hernia repair

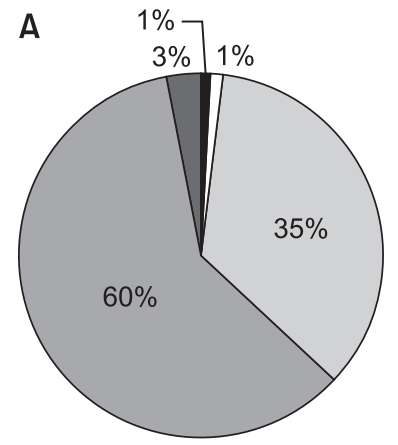

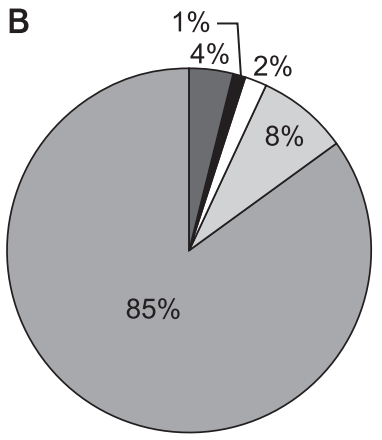

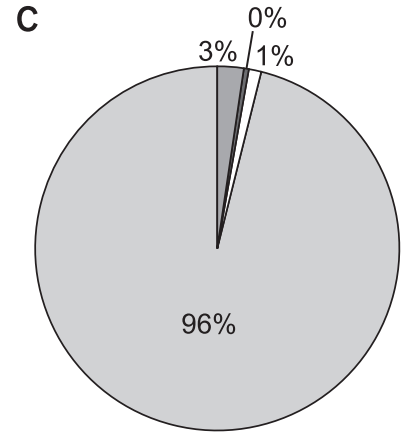

Fig. 6. Distribution of inguinal hernia repairs by procedures: $(\mathrm{A})$ total inguinal hernia repairs, (B) adult group, and (C) pediatric group. 


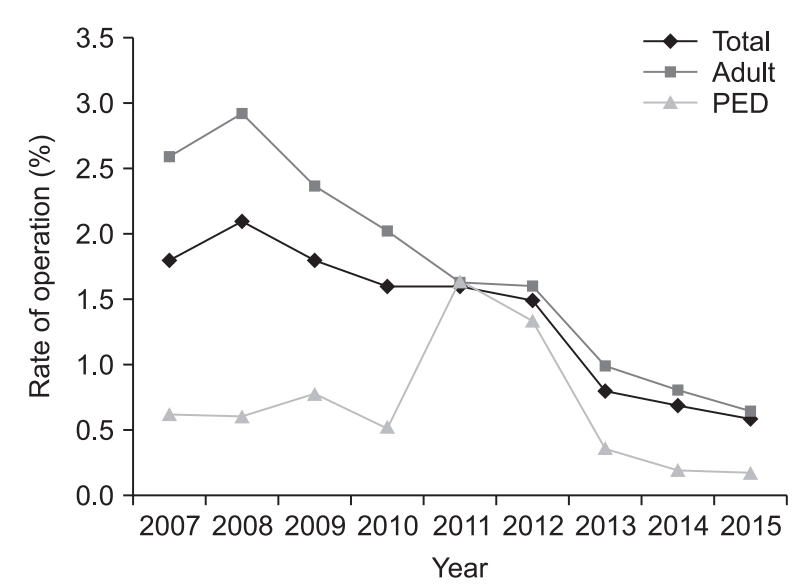

Fig. 7. Trend of proportion of inguinal hernia repair with bowel resection for 9 years. PED, pediatric patient.

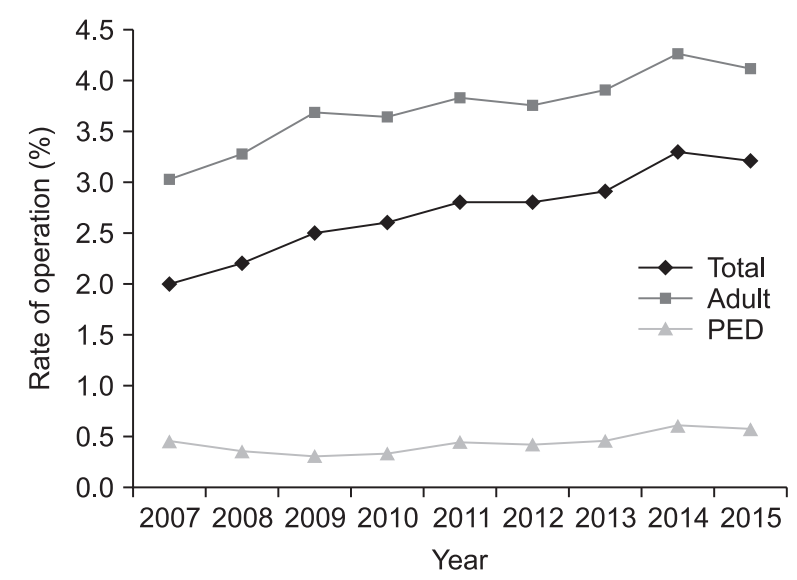

Fig. 8. Trend of proportion of recurrent inguinal hernia repair for 9 years. PED, pediatric patient.

between age groups (Fig. 6). High ligation and posterior repair procedures were the most common procedures for adults, while high ligation was the main procedure for pediatric herniorrhaphy. Few recurrences, femoral hernias, and bowel resections with herniorrhaphies were reported for children. The proportion of hernia repairs with strangulated intestine resection decreased from $1.8 \%$ to $0.6 \%$. This trend was also observed in adult patients (2.6\% in 2007 to $0.7 \%$ in 2015) (Fig. 7). However, although the proportion of recurrent inguinal hernia repairs was under $5 \%$ of total repairs, recurrent inguinal hernia repairs gradually increased from 596 repairs (2.0\%) in 2007 to 1,130 repairs (3.2\%) in 2015 (Fig. 8). The increasing pattern of recurrent inguinal hernia repairs was significantly higher in adults, from 535 repairs (3.0\%) in 2007 to 1,068 repairs (4.1\%) in 2015. Femoral hernias were rare in pediatric patients and typically constituted about $1 \%$ of the total (Fig. 9).

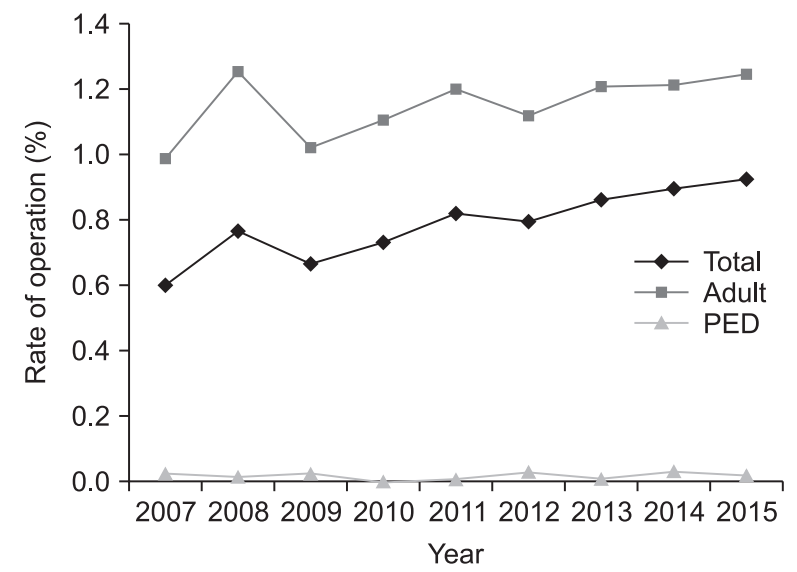

Fig. 9. Trend of proportion of femoral hernia repair for 9 years. PED, pediatric patient.

\section{DISCUSSION}

Inguinal hernia repairs are a commonly performed procedure in general surgery departments for both adults and children. Inguinal hernia repairs are performed worldwide, yet only a few studies are available on the prevalence of inguinal hernia, especially in Asia. This nationwide study provides largescale evidence for the prevalence of inguinal hernia repairs using data from the HIRA database in Korea. This study also investigated several nationwide characteristics of Korean inguinal hernia repairs associated with the change of the medical payment system to Korea's unique national health insurance program $(\mathrm{NHI})$.

The NHI achieved universal health coverage in 1989, and its coverage expanded to the whole population in 2000. The NHI finances the Republic of Korea's health system, and covers about $97 \%$ of the all Koreans. The remaining 3\% are covered by the Medical Aid program for low-income citizens. The Ministry of Health and Welfare plays a central role in health planning, policy formulation, and policy implementation. The Ministry of Health and Welfare has delegated the task of running the NHI to 2 quasi-public organizations - the National Health Insurance Service and the HIRA [4]. Formerly, every health-care provider requested payment from the HIRA for surgery after providing medical service and were paid under a fee-for-service system. However, this payment system changed to DRG-based prospective payments in 2002 for several disease categories, including inguinal hernia, to help prevent the rapid increase in health expenditures. The DRG system was expanded to entire hospitals in 2013 for seven disease categories: inguinal hernia, appendix, hemorrhoid, cataract, tonsil, and uterus surgery including Caesarean section.

Every year about 35,000 inguinal hernia repairs are performed in Korea. Numbers of repairs show a bimodal peak in prevalence at early childhood (1-5 years) and old age (50-79 
years). Our study confirmed the high prevalence (87\%) of inguinal hernia in males as reported in previous studies. However, the femoral hernia rate hernia repair rate (1\%) was lower than 3\% as reported in a previous study [5]. Additionally, costs gradually increased from $\$ 1,000$ to $\$ 1,600$ and a dramatic increase was observed in use of laparoscopic inguinal hernia repair from less than 3\% in 2007 to about 30\% in 2015. A similar increase in the use of laparoscopic surgery for pediatric patients $(0.07 \%$ in 2007 to $28.6 \%$ in 2015$)$ is also notable. Several European countries report an annual frequency of laparoscopic surgery that increased from $4.5 \%$ to $8 \%$ over six years. Laparoscopic repair was even more frequently performed in Korea [6].

The increase in use of laparoscopic surgery can be explained by several factors. First, easy access, wide availability of equipment, and standardized training for laparoscopic surgery encouraged increased use of the procedure. In Korea, laparoscopic surgery is widely in many surgical procedures including both simple laparoscopic cholecystectomy and appendectomy, and more challenging cases, such as colorectal surgery, gastrectomy and adrenalectomy. Nationwide data for Korea indicates that more than $60 \%$ of patients with colorectal cancer underwent laparoscopic surgery in 2013. This proportion of laparoscopic colorectal surgery is higher than reported for other countries; $41 \%$ in 2010 in the Netherlands, 44.8\% in 2012 in the United Kingdom, and 35.4\% in 2009 in the United States [7].

Second, the advantages of laparoscopic surgery encourage its greater use. In a previous study, laparoscopic inguinal hernia repair resulted in less postoperative pain, shorter hospital stays, and earlier return to work than open repair. Moreover, the incidence of recurrence was similar to incidence after open mesh repair [8].

Finally, a change in the medical insurance system might have led to an increased use of laparoscopic repair. Previous studies indicate that the costs for laparoscopic hernia surgery is greater than costs for open hernia surgery [9]. In Korea, the DRG based system was introduced in 2002 and expanded to all hospitals in 2013. When the government began the DRGbased system, inguinal hernia repair was included, and costs of repairs were fixed depending on surgical method. A higher cost for laparoscopic surgery for inguinal hernia was chosen compared with open surgery. As shown in Fig. 1, while annual inguinal hernia repairs remain about constant at 35,000 cases, medical costs increased steadily over the 9 years of the study. Use of laparoscopic surgery showed a similar increase over the same period. It seems reasonable to assume that higher medical cost reimbursement encouraged increased use of laparoscopic surgery. Hospitals can charge 1.6 times the cost of open surgery when using the laparoscopic procedure.

Some differences were noted between pediatric and adult inguinal hernia repairs. Several similarities were noted, such as male dominance, increasing use of laparoscopy, medical cost and decreased total hospital day, yet actual procedures used for repair were quite different. In children, femoral hernia, bowel resected inguinal hernia, and recurrent inguinal hernia repairs were rare, less than one percent. High ligation was the main procedure use for inguinal hernia repairs.

This difference probably reflects the different etiology of hernia in the 2 age groups. Overall, the etiology of inguinal hernias is still unclear, and it differs depending on ages, sex, and whether a hernia is direct or indirect. A patent processus vaginalis and increased cumulative abdominal pressure are risk factors for indirect inguinal hernias. Aging and altered connective tissue architecture and homeostasis resulting in weak abdominal walls are the risk factors for direct inguinal hernias [10]. Inguinal hernias in children are mostly indirect, probably due to the embryological failure of the obliteration of the processus vaginalis. Previous studies reported that patent processus vaginalis was more common in boys with inguinal hernias and the incidence is highest during the first year of life [11]. However, in this study, 1- to 5-year-old children had more frequent inguinal hernia repairs than did infants aged less than 1 year. This peak reflects the use of a larger age range for children than was used in the previous study. Moreover, the incidence of incarceration in pediatric inguinal hernia was previously reported to be 14 to 31 percent [12]. However, the rate of strangulated bowel resected inguinal hernia repairs in children was less than $1 \%$ in this study. In contrast, the etiology of adult inguinal hernia is multifactorial and may include genetic disorders, patent processus vaginalis, work load, and weakening connective tissue architecture due to aging. In this study, a second incidence peak was found in adult patients aged 50 to 70 . Bimodal peaks were also reported in studies based on nationwide data in Denmark [5].

Recurrence is a challenging aspect of inguinal hernia repair and it remains an unsolved problem, despite technical and socio-economic improvements. The recurrence rate for inguinal hernia ranges from $1 \%$ to $10 \%$ depending on surgical procedure, hernia type, surgeons' experience, and follow-up period [13]. Our study showed that, in Korea, every year approximately $2 \%-3 \%$ of repair operations are performed for recurrent hernia. To date, identified risk factors for recurrent inguinal hernia include controllable factors such as technical surgical methods, methods for anesthesia, mesh-fixation techniques, surgeon experience, and noncontrollable patient-related risk factors such as sex, hernia type, connective tissue composition and family disposition [14]. Restrictions in data provided by the HIRA for this study limit the analysis of these risk factors.

This study has some limitations and weaknesses. Data from the HIRA were used to define hernia prevalence, but these data do not reflect the true prevalence of inguinal hernia. Further, 
data from the HIRA do not include detailed information for patient and surgical outcomes, and it is difficult to compare groups and analyze risk factors for recurrence.

Nevertheless, this study is the first to report the nationwide prevalence of inguinal hernia surgery and the trend toward using more laparoscopic procedures for inguinal hernia repair in Korea. Further, we showed trends in surgical methods, total hospital stay, and medical costs since the DRG-based medical system was introduced. We suggest that the medical system might lead to changes in surgical trends.

\section{CONFLICTS OF INTEREST}

No potential conflict of interest relevant to this article was reported.

\section{REFERENCES}

1. Bay-Nielsen M, Kehlet H, Strand L, Malmstrom J, Andersen FH, Wara P, et al. Quality assessment of 26,304 herniorrhaphies in Denmark: a prospective nationwide study. Lancet 2001;358:11248.

2. Rutkow IM. Demographic and socioeconomic aspects of hernia repair in the United States in 2003. Surg Clin North Am 2003;83:1045-51.

3. Beard JH, Oresanya LB, Ohene-Yeboah M, Dicker RA, Harris HW. Characterizing the global burden of surgical disease: a method to estimate inguinal hernia epidemiology in Ghana. World J Surg 2013;37: 498-503.

4. Kwon S, Lee TJ, Kim CY. Republic of Korea health system review. Geneva (Switzerland): World Health Organization; 2015.

5. Burcharth J, Pedersen M, Bisgaard T, Pedersen C, Rosenberg J. Nationwide pre- valence of groin hernia repair. PLoS One 2013;8:e54367.

6. Wara P, Bay-Nielsen M, Juul P, Bendix J, Kehlet $\mathrm{H}$. Prospective nationwide analysis of laparoscopic versus Lichtenstein repair of inguinal hernia. Br J Surg 2005:92:127781.

7. Park SJ, Lee KY, Lee SH. Laparoscopic surgery for colorectal cancer in Korea: nationwide data from 2008-2013. J Minim Invasive Surg 2015;18:39-43.

8. McCormack K, Scott NW, Go PM, Ross S, Grant AM; EU Hernia Trialists Collaboration. Laparoscopic techniques versus open techniques for inguinal hernia repair. Cochrane Database Syst Rev 2003; (1):CD001785.

9. Eklund A, Carlsson P, Rosenblad A, Montgomery A, Bergkvist L, Rudberg C, et al. Long-term cost-minimization analysis comparing laparoscopic with open (Lichtenstein) inguinal hernia repair. Br J Surg
2010;97:765-71.

10. Oberg S, Andresen K, Rosenberg J. Etiology of inguinal hernias: a comprehensive review. Front Surg 2017;4:52.

11. Pan ML, Chang WP, Lee HC, Tsai HL, Liu CS, Liou DM, et al. A longitudinal cohort study of incidence rates of inguinal hernia repair in 0- to 6-year-old children. J Pediatr Surg 2013;48:2327-31.

12. Puri P, Guiney EJ, O'Donnell B. Inguinal hernia in infants: the fate of the testis following incarceration. J Pediatr Surg 1984; 19:44-6.

13. Burcharth J, Andresen K, Pommergaard HC, Bisgaard T, Rosenberg J. Recurrence patterns of direct and indirect inguinal hernias in a nationwide population in Denmark. Surgery 2014;155:173-7.

14. Burcharth J. The epidemiology and risk factors for recurrence after inguinal hernia surgery. Dan Med J 2014;61:B4846. 